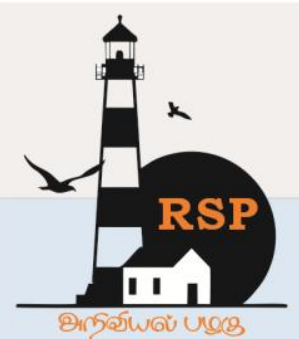

INTERNATIONAL RESEARCH JOURNAL ON ADVANCED SCIENCE HUB ISSN : $2582-4376$
Open Access

RSP SCIENCE HUB

(The Hub of Research Ideas)

Available online at www.rspsciencehub.com

\title{
A Review on the Relevance of Curcuminoids in Corona Pandemic Situation
}

Lovely Jacob $A^{1}$, Tom Cherian ${ }^{2}$

${ }^{1}$ Assistant Professor, Department of Chemistry, Little Flower College, Guruvayoor, Kerala, India

${ }^{2}$ Dr.Tom Cherian, Assistant Professor, PG and Research Department of Chemistry, Christ College

,Irinjalakuda, Kerala, India

lovely.a@littleflowercollege.edu.in ${ }^{1}$,drtomcherian@gmail.com ${ }^{2}$

\begin{abstract}
A hyperactive response by the immune system is said to be primarily responsible for pneumonia, also a fall out of a severe SARS-CoV-2 infection. Infection starts when the viral spike protein binds to its complementary host cell receptor. Viruses from the corona primarily attack epithelial cells. Depending on the species of the corona virus, they are transmitted from one host to another by either an aerosol, fomite, or faecal-oral route. Chloroquine and hydroxychloroquine are interferon blockers but they increase the risk of unsafe heart rhythm disturbances. Curcumin, a photochemical which occurs naturally in Curcuma longa, is known to have strong pharmacological properties. But it is essential to overcome the limitations for using it in medication. By docking, curcumin and its derivatives were shown to have the capacity to serve as spike protein inhibitors and thus viral entry. In this review, papers focused on structure, platforms and ill effects of Corona virus, and also discloses the nutrients which include mainly poly phenols and antioxidants containing curcuminoids which has antivirus and anti inflammatory properties; the nutrients under discussion can support the healthy functioning and strengthening of the respiratory system.
\end{abstract}

Key words: Corona pandemic, Curcuminoids, Antioxidants, Antivirus, Anti inflammatory, Molecular docking

\section{Introduction}

Owing to its high mortality, corona virus infection has gained significant attention in the last two decades. An unknown respiratory infection emerged from Wuhan, China has reported in late 2019. The source of this infection has been described as a novel corona virus associated with Extreme Acute Respiratory Syndrome ( SARS), which triggered 2002-2004 outbreaks and 2012 Middle East Respiratory Syndrome (MERS). By early March 2020, the new SARS-CoV-2 corona virus had infected more than 90,000 people worldwide and killed at least 3,100 people [1]. COVID-19 's key clinical sign is the development of respiratory symptoms but certain patients experience significant complications of the cardiovascular and the renal. The mechanism by which this virus induces problems to establish treatment options is urgently to be understood. According to the WHO, the most common diagnosis for severe COVID-19 is severe pneumonia [2]. A hyperactive response by the immune system is said to be primarily responsible for pneumonia, also a fall out of a severe SARSCoV-2 infection.

In this present scenario, we focused on papers which revealed the structure, platforms and ill effects of corona virus on human body. It has led us to look for different treatments in this field and find that there is no known successful pharmaceutical treatment as of this time, although it is much needed [3]. In multicenter clinical trials performed in China, chloroquine and hydroxy 
chloroquine have been shown to have clear efficacy and reasonable protection against COVID-19 associated pneumonia. These include direct antiviral effects, such as blocking steps required to replicate some viruses, and its ability to inhibit the development and release of proteins involved in many viral disease inflammatory complications. But the study's results are impressive. After correction for other risk factors, it appears that the incidence of ventricular arrhythmias was doubled in patients on hydroxychloroquine and tripled in chloroquine. When an antibiotic such as azithromycin was applied as proposed by some advocates, the risk sprang to fivefold.Here, we focused the antiviral and anti inflammatory properties of curcumin analogues. And it's relevance in medication during the Corona pandemic situation.

\section{Structure, platform and ill effects of corona virus}

The SARS-CoV-2 is an enveloped $\beta$ corona virus with a large, positive-sensitive, single-stranded RNA genome ranging from bases of 26 to $32 \mathrm{~kg}$. The four structural proteins present in the virus are the Spike (S) protein, envelope (E), membrane (M) protein, and nucleocapsid $(\mathrm{N})$ protein. The spike(S) protein interacts with the host cell membrane to allow virus entry during infection. Infection starts when the viral spike protein binds to its host cell receptor [4]. The corona virus reaches the human cells by binding its spike protein via the ACE2 (angiotensinconverting enzyme2) receptor. When attached, a host cell protease cleaves and activates the spike protein attached to the receptor [4]. Cleavage and activation, depending on the availability of host cell protease, enables the virus to reach the host cell by endocytosis or direct fusion of the viral envelope with the host membrane. The virus particle is uncoated upon entry into the host cell, and its genome reaches the cytoplasm of the cell [4]. Viruses may be transmitted into the atmosphere by the Contaminated Carriers. The corona virus spike protein 's association with its complementary cell receptor is crucial to deciding the released virus' tissue tropism, infectivity and variety of species. Corona viruses are predominantly aimed at epithelial cells. Depending on the species of corona virus, they are transmitted from one host to another through either an aerosol, fomite, or faecal-oral route. Recent work shows that SARS-CoV-2 spikes bind to receptors on the human cell surface called ACE2 like the virus that triggered the 2002 SARS outbreak. CoVs are zoonotic pathogens due to their high mutation rates that can affect different animals and humans resulting in a wide variety of clinical characteristics, ranging from asymptomatic course to multi-organ failure.

\section{Potential utility of curcuminoids in the battle of covid - 19}

Many virus-induced pneumonia studies have highlighted the possible use of curcumin in improving the lung index and survival rate. Up to now, curcumin has been believed to help human health and prevent diseases [5]. A recent study indicated that a low dose of curcumin $(80 \mathrm{mg} /$ day) was responsible for a number of healthpromoting acts, such as direct and indirect antioxidant activities [5]. Additionally, accumulating data from animal studies has shown that curcumin is preventing serious pneumonia from emerging. Thus, curcumin pretreatment (5 $\mathrm{mg} / \mathrm{kg} /$ day) prevents paraquat-induced lung inflammation and systemic lung remodelling at an early stage of ALI (Acute Lung Infection). Lai et al. (2020) stated that curcumin administration during pre-infection or post-infection significantly increases the lung index and prolongs the survival rate. Interestingly enough, pre-administration with curcuma often decreases the fatality risk [6]. All these findings indicate that curcumin administration may have both prophylactic and therapeutic effects on virus-induced pneumonia and mortality. Some clinical trials have documented beneficial effects in the treatment of cardiovascular diseases, metabolic syndrome or diabetes, and infectious diseases, especially viral infections. All these findings reveal that curcumin reduces these diseases mainly through immune response modulation.

Several polyphenolic compounds derived from natural products were established in the sense of preventive and supportive therapy with varied antiviral mechanisms such as targeting virus specific interactions, viral entry, replication, and assembly. In accordance with these observations, curcumin is one of the natural compounds commonly researched for its antiviral effects [7]. The development of pneumonia evoked by 
coronavirus is associated with excessive inflammatory responses in the lung, known as "cytokine storms," resulting in pulmonary edoema, atelectasis and acute lung injury (ALI) or fatal acute respiratory distress syndrome (ARDS). Even now, there are no appropriate treatments available for excessive immune response-mediated lung injury suppression. It is possible to speculate that curcumin may be used as a therapeutic drug for viral pneumonia and ALI / ARDS, given the low toxicity and its antioxidant, anti-inflammatory, and antiviral activities.

\subsection{Curcumin as an antiviral agent}

Many studies have reported that curcumin interferes with the process of viral infection through multiple mechanisms, including direct targeting of viral proteins, inhibiting particle development and gene expression, and blocking virus entry, replication and budding. A recent in vitro research showed that curcumin inhibits respiratory syndrome virus (RSV) by blocking attachment to host cells. In another research with IAV (influenza A virus) infected cells, it was found that curcumin inactivates different IAV strains directly, disturbs their adsorption and inhibits their replication[8]. Curcumin's antiviral effects on viruses have been reported, including vesicular stomatitis virus, par influenza virus type 3 , vesicular stomatitis virus, flock house virus, herpes simplex virus, and respiratory syncytial virus[9]. Curcumin treatment has been shown to be capable of altering the surface protein structure in viruses, thus preventing virus entry and virus budding.Accordingly, an analysis of 221 phytocompounds anti-SARS-CoV activity showed that $20 \mu \mathrm{M}$ of curcumin exhibits major inhibitory effects in a cell-based cytopathogenic effect (CPE) assay by Vero E6. The authors provided evidence of a mild effect of curcumin against replication of SARS-CoV and the inhibitory effect of curcumin on protease activity of SARS-CoV 3CL which is important for replication of SARS-CoV.

\subsection{Curcumin may theoretically target crucial} steps in the cycle of viral replication

The virus uses cellular machinery to regulate and replicate its metabolic processes. The antiviral agents can prevent virus growth in infected cells without destroying the healthy cells. Curcumin modulates intercellular signalling cascades that are necessary for efficient virus replication such as attenuation of NF- $\pi \mathrm{B}$ (Nuclear Factor kappa-light-chain-enhancer of activated $\mathrm{B}$ cells) and PI3K / Akt (Phosphatidylinositol 3kinase and protein kinase B) signalling It also affects post-transcription and post-translation cellular modifications, thus restricting viral propagation by interfering with essential steps in their replication cycle, including genome replication, and viral attachment[10]. Attachment, penetration, uncoating, and replication of the genome are some of the virus replication process. Some of the known effects of curcumin include preventing viral infection by targeting virus penetration and disrupting viral replication components[11]. When curcumin was added to cells before or after infection, some viruses, enveloped viruses, including poxvirus, flavivirus, herpes virus, and orthomyxovirus, were attenuated. [12]. Curcumin can alter the structure of the surface protein in viruses and block viruses from entering the cell. In addition, on the surface, the positively charged curcumin undergoes electrostatic interactions with cell membranes and competes with the virus to bind with the cells.

\subsection{Curcumin has potential effects on interferons}

Potential anti-coronaviral therapies target the human or virus cells themselves. Human immune system is considered to play a significant role in destroying the virus and studies have shown the type I and type II interferons antiviral activity. Interferon-beta (IFN- $\beta$ ) has been documented as reducing MERS-CoV in vitro replication[13]. The other targets in human cells are blocking the cell surface receptors for binding the corona virus and the cell signalling pathways that aid in viral replication. Angiotensin-converting enzyme 2 (ACE2) is one of the candidates proposed for targeting target drug therapy to prevent virus infection because the virus enters the cell through ACE2 receptors[14]. Passive antibody therapy can be considered one of the possible strategies for reducing the pandemic COVID-19. These are preliminary results, however, and none of these agents have yet to be licenced for therapeutic use in the management of COVID-19 infected patients [15].

3.4 Curcumin's possible role in treating
pulmonary inflammation and edema 


\subsubsection{Pro inflammatory cytokine control}

Curcumin blocks the critical signals that control the expression of various pathways for proinflammatory cytokines including the nuclear factor- $\pi \mathrm{B}$ and MAPK (mitogen-activated kinase protein). Curcumin has anti-inflammatory and anti-fibrotic effects by reducing lung-involved expression of essential chemokines and cytokines. Inflammation plays a key role in pathogenesis of viral infection lung complications as demonstrated by lung edoema, haemorrhage, invasion of neutrophils and alveolar thickening. Studies show that curcumin and its analogues are able to ameliorate lung damage.Infiltration of polymorphonuclear neutrophils (PMNs) is associated with pulmonary edoema and may release oxidants and proteases that damage the alveolar-capillary membrane and cause plasma proteins to leak out of the blood vessels, thus causing pulmonary edoema[16]. More recently, Xiao et al. [17]reported that curcumin analogue C66 prevents lipopolysaccharide (LPS)-induced ALI by suppressing the JNK pathway (Jun aminoterminal kinases) and subsequently inhibiting the expression of inflammatory cytokines. Cytokine storm is triggered by numerous causes that may emerge from autoimmune, inflammatory, iatrogenic, and infectious origins[18]. It is characterised by the development of excessive quantities of inflammatory cytokines induced by unregulated feed forward activation and immune cell amplification. Infections from viruses such as Ebola, avian influenza, dengue, and coronavirus can contribute to cytokine storms, which can create a huge amount of pro-inflammatory cytokines. The concerted activity of these inflammatory mediators induces tissue and cell death, which is characterised by clinical syndromes such as severe pulmonary edoema, alveolar haemorrhage, ARDS and multiple organ failures [19]. There is clear evidence from patients infected with the corona virus with both high levels of cytokine and lung pathological changes[20]. Numerous studies in vivo and in vitro have shown that curcumin and its analogues inhibit markedly the synthesis and release of proinflammatory cytokines.Direct pulmonary delivery of solubilised curcumin is found to significantly decrease pro-inflammatory cytokines. The mechanism underlying inflammation regulation of curcumin has been extensively investigated and involves numerous signalling pathways, in which NF-ÿB plays a key role.

\subsubsection{Regulation of anti-inflammatory cytokines}

Curcumin has been shown to positively regulate anti-inflammatory cytokines, in particular IL-10(Interleukin 10). Curcumin can therefore serve as a double-edged sword in the sense of inflammation, decreasing the regulation of proinflammatory cytokines, and upregulating antiinflammatory IL-10.Curcumin has also been controlled other inflammatory mediators. Curcumin treatment is found to effectively inhibit the degradation of $\mathrm{Iy} B \alpha$ and disrupt the development of COX-2 in an animal model of chronic obstructive lung disease [21]. Curcumin inhibits the virus-induced expression of the genes TLR2/4/7, MyD88, TRIF, and TRAF6 and blocks the IAV-induced phosphorylation of Akt, p38, JNK as [4] in addition to disrupting the pathway to NF-kB.

\subsection{Curcumin does away with fibrosis}

Pulmonary fibrosis also affects the ALI after the viral infection which can lead to death. Curcumin has been documented to be able to suppress pulmonary fibrosis. In the model of lung damage caused by radiation, cytokine accumulation and collagen deposition occur in the interstitial space, in parallel with lung tissue fibrosis[19]. Curcumin prevents the penetration of macrophages and lymphocytes, and enhances fibrosis[19]. In pulmonary fibrosis studies with bleomycin / SiO2 / amiodarone, curcumin has also been shown to directly reduce the expression of TGF- $\beta$ protein and its mRNA[4].

\subsection{Curcumin's possible role in inhibiting oxidative stress in viral infection}

Curcumin has the capacity of electron transfer to scavenge various small oxidative intracellular molecules[22]. Curcumin can increase glutathione ( $\mathrm{GSH}$ ) expression and inhibit reactive oxygen species ( ROS) and malondialdehyde ( MDA) generation. [23].

\section{Molecular Docking Studies}

Curcumin has less bioavailability. Thus, direct consumption of curcumin does not contribute to the health benefits associated with it.This is due to it's poor absorption, rapid metabolism, and rapid elimination. Several clinical studies have shown that the question of curcumin 


\section{www.rspsciencehub.com}

bioavailability can be mitigated by administering higher concentrations within non-toxic limits[24]. To improve bioavailability, structural homologues involving modification of all the groups present in curcumin were prepared. The literatures identify various analogues of synthetic curcumin with a broad range of applications. Furthermore, several studies have suggested different ways of enhancing the bioavailability of curcumin, such as processing and encapsulation of curcumin into micelles, liposomes, phospholipid complexes, exosomes or polymeric nanocarrier formulation and also the use of curcumin in conjunction with cellulosic derivatives, natural antioxidants and a hydrophilic carrier[25].

The precursors needed for the synthesis of curcumin analogues is very costly and the purification, separation processes are time consuming. More over that, after synthesizing curcumin analogues and complexes, they may not be possess the expecting biological activities also. So, by depending up on computational chemistry such as Molecular Docking by which, we can predict better interactions of ligands with targeting molecules and some of their biological activities. Computational methods are the scientists' first and foremost preference for prophecy of apparent binding ligand modes and affinities prior to experimental studies. Additionally, in recent years, improving speed, reliability and accuracy of computational docking methods has made it a suitable option to develop structure-based drugs. Recently, a molecular ducking analysis has shown that curcumin has better receptor binding potential and can prevent Covid-19 virus infection. Spike glycoprotein is a possible drug targeting candidate for inhibiting virus entry[26]. Studies of silico docking showed that curcumin could theoretically inhibit ACE2 to prevent the cell 's entry into Covid-19. One of the possible therapeutic strategies for virus inhibition is focused on the use of agents that could inhibit replication[18]. In addition, Ting $\mathrm{Du}$ et al. researched curcumin's effects on the synthesis of negative-strand RNA by using PEDV (Porcene epidemic diarrhoea virus) as a model of corona virus. The evidence supports curcumin's potential position as a promising antiviral agent. Utomo et al. used target receptor molecular docking, including SARS-CoV-2 protease, spike glycoprotein- RBD, and PD-ACE2,
Volume 02 Issue 08 August 2020

which are assumed to participate in virus infection as a guide compared to the recognised ligand or drugs.

\section{Conclusion}

In different forms of virus infections, the multi-mechanistic modes of action are the powerful of curcumin. The paper cites several studies in which curcumin in pulmonary inflammation, fibrosis, and oedema is successful. It also addresses the positive function of curcumin played during viral infections under the cytokine storm conditions. It is clear from now on that the biological properties including the advance mode of drug delivery of curcumin could be considered when formulating the pharmaceutical products and their application as a preventive measure in the inhibition of human transmission of SARS-COV2 infection. In conclusion, we suggest that curcumin may be used in any clinical setting as a supportive therapy for the treatment of Covid-19 disease to counteract the lethal effects of SARS-CoV-2.

\section{References}

[1].https:/www.nih.gov/news-events/nihresearch-matters/novel-coronavirusstructure-revelations-targets-vaccinestreatments, Latest corona virus structure reveals vaccine and treatment goals, March 3, 2020

[2].https:/www.who.int/news-room/q-adetail/q-a-coronaviruses,"Covid-19 (Corona Virus Disease 2019) is an international public health emergency.

[3]. Cortegiani, Ingoglia, Ippolito, Journal of Critical Care, "A systematic review on the efficacy and safety of chloroquine for the treatment of COVID-19", Vol 57, Pg.279283, June 1,2020

[4].https://en.wikipedia.org/wiki/Coronavirus

[5].DiSilvestro, R. A., Joseph, E., Bomser, J., Zhao, S. (2012).(2012). Various effects of a low-dose lipidated curcumin supplement in stable middle aged people Nutr. 11:79, J.Vol 11.

[6].Y., Yan, Y., Liao, S., Li, Y., Ye, Y., Liu, N., and others (2020). 3D-quantitative link of structure-activity and antiviral effects of curcumin derivatives as potent inhibitors of H1N1 neuraminidase influenza. Arch Pharm Res. 
[7]. Es. Das S, Sarmah S, Lyndem S, Roy AS. An inquiry into possible SARS-CoV-2 key protease inhibitors using molecular docking studies. J Biomol Dyn schema. Europe 2020;13:1-11.

[8].Dai, J., Gu, L., Su, Y., Wang, Q., Zhao, Y., Chen, X., et al. (2018). Inhibition of curcumin on influenza A virus infection and influenzal pneumonia via oxidative stress, TLR2/4, p38/JNK MAPK and NF$\kappa \mathrm{B}$ pathways. Int. Immunopharmacol. 54, 177-187.

[9].Zorofchian Moghadamtousi, S., Abdul Kadir, H., Hassandarvish, P., Tajik, H., Abubakar, S., \& Zandi, K. (2014). A review on antibacterial, antiviral, and antifungal activity of curcumin. BioMed Research International, 2014, 186864.

[10].Praditya, D., Kirchhoff, L., Brüning, J., Rachmawati, H., Steinmann, J., \& Steinmann, E. (2019). Anti-infective properties of the golden spice curcumin. Frontiers

in

Microbiology, 10, 912.

[11].Mathew, D., \& Hsu, W.-L. (2018). Antiviral potential of curcumin. Journal of functional foods, 40, 692- 699.

[12].Chen, T.-Y., Chen, D.-Y., Wen, H.-W., Ou, J.-L., Chiou, S.-S., Chen, J.-M., .Hsu, W.-L. (2013). Inhibition of enveloped viruses infectivity by curcumin. PloS one, $8(5)$.

[13].Hui, D. S., Azhar, E. I., Madani, T. A., Ntoumi, F., Kock, R., Dar, O., Drosten, C. (2020). The continuing 2019-nCoV epidemic threat of novel corona viruses to global health-The latest 2019 novel coronavirus outbreak in Wuhan, China. International Journal of Infectious Diseases, 91, 264- 266.

[14].Xu, H., Zhong, L., Deng, J., Peng, J., Dan, H., Zeng, X., Chen, Q. (2020). High expression of ACE2 receptor of 2019-nCoV on the epithelial cells of oral mucosa. International Journal of Oral Science, 12(1), 1- 5 .

[15].Yan, R., Zhang, Y., Li, Y., Xia, L., Guo, Y., \& Zhou, Q. (2020). Structural basis for the recognition of SARS-CoV-2 by full-length

human

ACE2. Science. 367(6485), 1444- 1448.

[16].Matthay, M. A., and Zimmerman, G. A. (2005). Acute lung injury and the acute respiratory distress syndrome: four decades of inquiry into pathogenesis and rational management. Am. J. Respir. Cell Mol. Biol. 33, 319-327.

[17].Xiao, Z., Xu, F., Zhu, X., Bai, B., Guo, L., Liang, G., et al. (2019). Inhibition Of JNK phosphorylation by curcumin analog C66 Protects LPS-induced acute lung injury. Drug Des. Dev. Ther. 13, 41614171.

[18].Behrens, E. M., and Koretzky, G. A. (2017). Cytokine storm syndrome: looking toward the precision medicine era. Arthritis Rheumatol. 69, 1135-1143.

[19].Amini, P., Saffar, H., Nourani, M. R., Motevaseli, E., Najafi, M., Ali Taheri, R., et al. (2018). Curcumin mitigates radiationinduced lung pneumonitis and fibrosis in rats. Int. J. Mol. Cell. Med. 7, 212-219.

[20].Channappanavar, R., and Perlman, S. (2017). "Pathogenic human coronavirus infections: causes and consequences of cytokine storm and immunopathology. Semin. Immunopathol. 39, 529-539.

[21].Yuan, J., Liu, R., Ma, Y., Zhang, Z., and Xie, Z. (2018). Curcumin attenuates airway inflammation and airway remolding by inhibiting NF- $\kappa \mathrm{B}$ signaling and $\mathrm{COX}-2$ in cigarette smoke-induced COPD mice. Inflammation 41, 1804-1814.

[22].Barzegar, A., \& Moosavi-Movahedi, A. A. (2011). Intracellular ROS protection efficiency and free radical-scavenging activity of curcumin. PLoS One, 6(10), e26012.

[23].Rong, S., Zhao, Y., Bao, W., Xiao, X., Wang, D., Nussler, A. K., Liu, L. (2012). Curcumin prevents chronic alcohol-induced liver disease involving decreasing ROS generation and enhancing antioxidative capacity. Phytomedicine, 19(6), 545- 550.

[24].Kunnumakkara, A. B., Harsha, C., Banik, K., Vikkurthi, R., Sailo, B. L., Bordoloi, D., Aggarwal, B. B. (2019). Is curcumin 
bioavailability a problem in humans: Lessons from clinical trials. Expert Opinion on Drug Metabolism \& Toxicology, 15(9), 705- 733.

[25].Jäger, R., Lowery, R. P., Calvanese, A. V., Joy, J. M., Purpura, M., \& Wilson, J. M. (2014). Comparative absorption of curcumin formulations. Nutrition Journal, 13, 11.

[26].Utomo, R. Y., \& Meiyanto, E. (2020). Revealing the potency of citrus and galangal constituents to Halt SARS-CoV-2 infection. Preprints, 2020030214. 